\title{
Stacking fault structure in shear-induced colloidal crystallization
}

\author{
Tesfu Solomon and Michael J. Solomon ${ }^{\text {a) }}$ \\ Department of Chemical Engineering, University of Michigan, Ann Arbor, Michigan 48109
}

(Received 22 November 2005; accepted 30 January 2006; published online 5 April 2006)

\begin{abstract}
We report measurements of the spatial distribution of stacking faults in colloidal crystals formed by means of an oscillatory shear field at a particle volume fraction of $52 \%$ in a system where the pair potential interactions are mildly repulsive. Stacking faults are directly visualized via confocal laser scanning microscopy. Consistent with previous scattering studies, shear orders the initially amorphous colloids into close-packed planes parallel to the shearing surface. Upon increasing the strain amplitude, the close-packed direction of the (111) crystal plane shifts from an orientation parallel to the vorticity direction to parallel the flow direction. The quality of the layer ordering, as characterized by the mean stacking parameter, decreases with strain amplitude. In addition, we directly observe the three-dimensional structure of stacking faults in sheared crystals. We observe and quantify spatial heterogeneity in the stacking fault arrangement in both the flow-vorticity plane and the gradient direction, particularly at high strain amplitudes $(\gamma \geqslant 3)$. At these conditions, layer ordering persists in the flow-vorticity plane only over scales of $\sim 5-10$ particle diameters. This heterogeneity is one component of the random layer ordering deduced from previous scattering studies. In addition, in the gradient direction, the stacking registry shows that crystals with intermediate global mean stacking probability are comprised of short sequences of face-centered cubic and hexagonal close-packed layers with a stacking that includes a component that is nonrandom and alternating in character. (C) 2006 American Institute of Physics.
\end{abstract}

[DOI: $10.1063 / 1.2178784]$

\section{INTRODUCTION}

Under certain conditions of interaction potential and volume fraction, colloidal particles form equilibrium phases with three-dimensional positional order. ${ }^{1}$ Such colloidal crystals are a possible route to fabricate macroscopic structures with applications in photonics, ${ }^{2}$ sensors, ${ }^{3}$ and catalysis. ${ }^{4} \mathrm{Col}-$ loidal dispersions are also a fruitful model system to address fundamental questions of statistical mechanics related to crystallization, gelation, and the glass transition. ${ }^{5-7}$ Inherent in the natural ordering process of colloidal crystallization is the formation of defect structures such as stacking faults, grain boundaries, and vacancies. ${ }^{8-10}$ By reducing the degree of ordering, such defects compromise the periodicity that is necessary for the functionality of these materials. ${ }^{11,12}$ The application of external fields such as shear, gravity, or an electric field to direct this crystallization is one avenue to minimize defect concentration and manufacture crystals of high quality on large scales. ${ }^{13,14}$ In addition, this deficiency could possibly be overcome by combining postprocessing methods such as the use of optical gradient forces that have been shown to anneal small defect-laden regions in thin crystals. ${ }^{15}$

While some aspects of colloidal and molecular crystallization are analogous, polydispersity, sedimentation, finite size, and boundary effects as well as the small elastic constant of colloidal crystals may contribute to a higher defect concentration in colloidal systems. ${ }^{9,16,17}$ Moreover, the relatively small free energy cost associated with the formation of

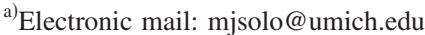

stacking faults make these defects a common feature in colloidal crystals with close-packed structure. ${ }^{18}$ Computational studies have shown that a face-centered cubic (fcc) crystal is entropically favored at equilibrium over a hexagonally closepacked (hcp) crystal but only by the relatively small amount of $\sim 10^{-4} k_{b} T$ per sphere. Simulations have also probed the correlation between neighboring crystal layers and identified a small entropic interaction that decreases with increasing layer separation. ${ }^{19}$ In addition to simulations, a range of faulted structures have been experimentally observed for unperturbed equilibrium crystals. ${ }^{20-23}$ However, the degree of correlation between stacking faults as well as the effect of external forces on the number and distribution of stacking faults $^{24,25}$ are poorly understood.

Scattering studies have probed the effect of flow on the nonequilibrium microstructure of colloidal crystals; however, few have discussed the effect of flow on defect composition since defect structures are poorly resolved by measurements such as scattering which probe ensemble averaged structure. Application of a fixed shear stress to colloidal dispersions generates a rich sequence of microstructural transformations as the system is increasingly perturbed from equilibrium. ${ }^{26,27}$ Oscillatory shear flow also generates metastable ordered structures from colloidal suspensions with amorphous equilibrium structure. ${ }^{28}$ Ackerson describes a complex succession of two-dimensional (2D) and three-dimensional (3D) structures realized by progressive variation of the applied shear field strength. ${ }^{29}$ A recent review of scattering studies of shear-induced colloidal crystallization is available by Vermant and Solomon. ${ }^{30}$ It is clear that the prevalence of defects 
in this succession of previously identified transitions warrants characterization. In this paper we focus on stacking fault defects.

To explain the microstructures observed in these sheared colloidal crystals at high volume fractions $(\sim>50 \%)$, Ackerson has proposed a mechanical model that accounts for the variation in the strain amplitude and volume fraction of the particles. ${ }^{29}$ At low strain amplitudes, particles are translated less than one particle diameter and thus remain in their respective interstitial sites during shearing. In this scenario, oscillating twinned fcc crystals with particles traveling in a zigzaglike trajectory allow for the least constrained deformation of the crystals. At large strain amplitudes, particles are driven more than one particle diameter. Subsequent particle interactions lead to a reorganization of the microstructure into a randomly stacked sliding layer configuration. At intermediate strain amplitudes a mixture of both microstructures and deformation mechanisms is possible. At progressively higher strain amplitudes and shear rates a number of different microstructure and deformation mechanisms have been observed, including shear melting. ${ }^{26,29}$

To date, colloidal crystallization has primarily been studied using scattering techniques. ${ }^{30}$ Such measurements, for example, deduce the unit cell and average stacking probability from the intensity modulation of the diffraction patterns arising from the crystal's periodicity. However, such analysis is limited to evaluating the global, ensemble averaged structure. For example, although the ratio of fcc to hcp layering can be extracted from scattering by computing the mean stacking parameter, details regarding the spatial distribution of stacking faults cannot. (Here the mean stacking parameter measures the probability of observing crystal translation vectors that yield a fcc or hep layer with $\alpha=0, \alpha=0.5$, and $\alpha$ $=1$ corresponding to complete hcp, random stacking, and pure fcc stacking probabilities, respectively.) Moreover, layer ordering is not uniquely determined by the mean stacking parameter. That is, two different layer structures (each with possibly distinct potential for successful application) can yield the same mean stacking parameter. For example, computer simulation has shown that colloidal crystals can nucleate from a twinned fcc structure that has an average stacking parameter equivalent to that for a random stacking sequence. ${ }^{24}$ This finding suggests that studies aimed at discovering the underlying mechanisms of defect formation and persistence in colloidal crystals are needed to resolve microstructural features with greater detail than the average measures yielded by scattering.

Direct visualization methods such as optical and electron microscopies complement the ensemble averaged characterization of scattering in this way. Confocal optical microscopy, in particular, can be applied to interrogate the $3 \mathrm{D}$ real space structure in solvents that is typical of colloidal crystallization. $^{31-33}$ These methods, when combined with quantitative image processing, allow the structure and dynamics of colloidal suspensions to be studied directly in real space. For example, visualization of specific crystallographic planes (110) within a colloidal crystal and the location of stacking faults have been observed. ${ }^{34}$ Defect structures, such as stringlike voids in colloidal suspensions sheared in confinement, ${ }^{35}$ may also be studied.

In this paper, we investigate the effect of oscillatory flow on the stacking registry of shear-induced colloidal crystallization. As reviewed in Ref. 30, a rich sequence of structural transitions (including, e.g., fully crystalline layers, polycrystals, randomly stacked layers, strings, and shear melted structures) have been previously observed. Among the most intriguing observations to date is that intermediate strain amplitudes and strain rates lead to scattering that is consistent with a random configuration of stacking registries. Here, we explore this possibility further by means of direct visualization methods. We use an oscillatory flow field so as to independently control the strain amplitude and deformation rate and thereby compare results with prior scattering results. While colloidal suspensions do crystallize under equilibrium conditions, we perform measurements at Peclet number Pe $>1$ to better understand a regime of nonequilibrium crystallization in which high quality crystals have previously been reported. ${ }^{36}$ Here Pe measures the relative effect of shear to thermal forces in the crystallization process. Our materials are concentrated suspensions of sterically stabilized poly (methyl methacrylate) (PMMA) colloids in nonpolar organic solvents. Due to approximate refractive index matching, attractive interactions between the particles are weak. The addition of disassociating salts also allows tuning of a repulsive component of the interaction potential into a regime that promotes colloidal crystallization. ${ }^{37}$

\section{EXPERIMENT}

\section{A. Colloid synthesis}

Monodisperse poly(methyl methacrylate) spheres were synthesized by adaptation of the methods of Antl et al., Campbell and Bartlett, and Pathmamanoharan et al. ${ }^{38-40}$ The particle diameter characterized by scanning electron microscopy was $1.15 \mu \mathrm{m}$ (standard deviation of $4.1 \%$ ). Briefly, the synthesis is a dispersion polymerization reaction of MM (Fluka) and methacrylic acid (Sigma). The synthesized particles were sterically stabilized by a graft layer of poly(12hydroxy-stearic acid) (PHSA) that was covalently bound to the surface through a locking step. The stabilizer was formed in a separate reaction of glycidyl methacrylate, PHSA, and $\mathrm{MM}$ in which product formation was verified by means of acid value titration and gel permeation chromatography. The fluorescent species, Nile red dye $\left(\lambda_{\mathrm{ex}}=525-605 \mathrm{~nm}\right.$, Sigma), was directly incorporated into the particles during the dispersion polymerization to allow for confocal fluorescence imaging. This dye was selected for its thermal stability and low rate of photobleaching. Particles were suspended in a highly viscous, nearly refractive index matched solvent of dioctyl phthalate (DOP) (Sigma). ${ }^{41}$ The refractive index matching solvent $(n=1.486)$ ameliorates the effect of attractive van der Waals forces on colloidal stability. The high viscosity of DOP minimizes Brownian displacements and sedimentation during confocal laser scanning microscopy (CLSM) image volume acquisition. Samples were prepared by dispersing dried PMMA in a mixture of DOP and tetrabutylammonium 


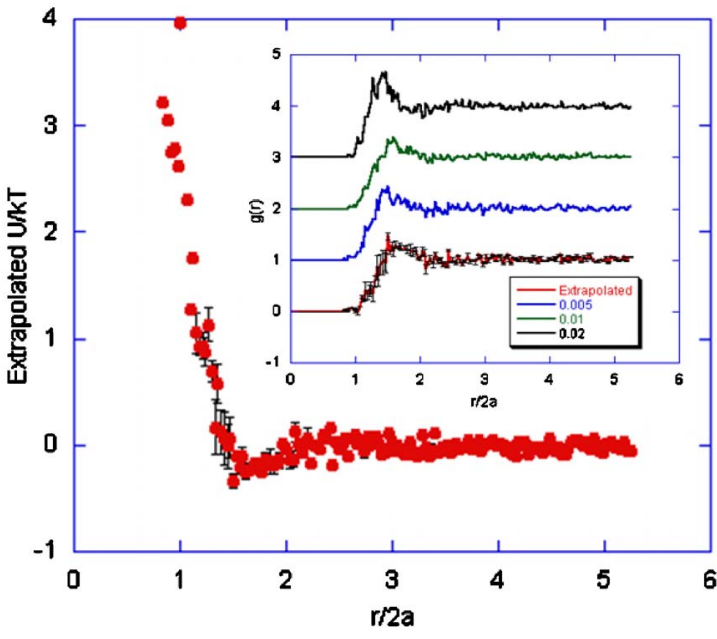

FIG. 1. Extrapolated dilute pair interaction potential. The inset is a dilute pair correlation function at several varying volume fractions.

chloride salt. ${ }^{37}$ The volume fraction of particles was $\phi$ $=0.52$ and the salt concentration was $10^{-3} \mathrm{M}$. The high volume fraction was selected to probe a regime where closepacked ordering of the suspensions is expected.

The pair potential $U(r)$ plotted in Fig. 1 was measured to assess the particle pair interactions. Briefly, we measured $g(r)$ at a number of dilute volume fractions and then applied the extrapolation

$$
\frac{U(r)}{k T}=\lim _{\phi \rightarrow 0}-[\ln g(r)]
$$

to obtain the colloid pair potential interaction $U(r)$. Measurements of the pair correlation function were taken at volume fractions ranging from $0.5 \%$ to $2 \%$. Results shown are the average of five experiments with the standard error of the mean reported as the error bars. Figure 1 shows a slight deviation from hard sphere behavior towards a mildly repulsive particle pair interaction of the order $\sim 1.3 d$ where $d$ is the particle diameter. In addition, a weak attraction is also present (order $\sim 0.5 k_{b} T$ ) consistent with the conclusions of other work on PMMA-PHSA colloids in organic solvents. ${ }^{42}$ The precision is estimated to be $\sim \pm 10 \%$. This estimate of the pair potential range is based on the precision of $3 \mathrm{D}$ image processing algorithms and the effect of the polydispersity of the colloids. ${ }^{43,44}$

\section{B. Shear flow}

An illustration of the shear flow cell used is in Fig. 2.

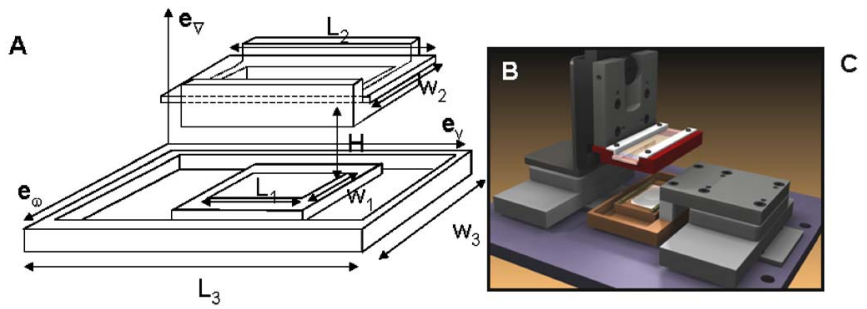

FIG. 2. Shear cell illustrations. (a) Schematic of shearing surfaces. (b) 3D rendering of shear cell components. (c) Table of dimensions.
TABLE I. List of primary shear cell dimensions. Definition of terms: $e_{\omega}, e_{\nabla}$, and $e_{v}$ represent vorticity, gradient, and velocity directions, respectively.

\begin{tabular}{cc}
\hline \hline$H$ & $150(\mu \mathrm{m})$ \\
\hline$L_{1}$ & $50(\mathrm{~mm})$ \\
$L_{2}$ & $10(\mathrm{~cm})$ \\
$L_{3}$ & $13(\mathrm{~cm})$ \\
$W_{1}$ & $24(\mathrm{~mm})$ \\
$W_{2}$ & $3(\mathrm{~cm})$ \\
$W_{3}$ & $7(\mathrm{~cm})$ \\
\hline \hline
\end{tabular}

Figure 2(a) provides an enlargement of the shearing surfaces with appropriate dimensions listed in Table I. Figure 2(b) is a $3 \mathrm{D}$ rendering which shows how the various components of the shear cell are integrated into the setup. The flow cell utilizes a parallel plate geometry with one surface a glass coverslip (Corning, PA) and the other a glass slide (Kineoptics, LA) to generate a homogeneous shear flow. Specimens are viewed from below through the glass coverslip (thickness of No. 1.5). Two tilt goniometers (gon40, Newport, CA) are aligned to achieve parallelism of $\pm 10 \mu \mathrm{m}$ over the coverslip area $\left(24 \times 50 \mathrm{~mm}^{2}\right)$. The alignment is performed by measuring the height of PMMA particles fused to the outer edge of the coverslip. The shear apparatus is mounted on a manual $x-y$ translational stage for variable positioning of the sample relative to the microscope objective. An oscillatory, plane Couette flow with a sinusoidal wave form is produced with a linear stepper motor (Labmotion, Coherent, CA). Sample volumes of $\sim 400 \mu \mathrm{l}$ were well mixed by vigorous vortex stirring and then placed between the glass surfaces by micropipetting. A linear micrometer (model 433, Newport, CA) was used to precisely adjust the gap height to $150 \mu \mathrm{m}$. By varying the strain amplitude and frequency (hertz), a range of shear rate magnitudes $\left(\mathrm{s}^{-1}\right)$, strain amplitudes, and Peclet numbers are accessible $(0.01<\dot{\gamma}<100,0.05<\gamma<23$, and $0.1<\mathrm{Pe}<50$, here $\mathrm{Pe}$ is computed from the free particle diffusivity). Strain amplitudes, shear rate magnitudes, and Peclet numbers realized during this study were $1<\gamma<4$, $0.25<\dot{\gamma}<4$, and $6.5<\mathrm{Pe}<19.5$. Constant shear rate magnitude experiments $\left(\dot{\gamma}=4 \mathrm{~s}^{-1}\right)$ were conducted by varying the strain amplitude with $1<\gamma<4$ with a proportional change in the frequency range of $4<$ Freq $(\mathrm{Hz})<1$. In order to expose samples sheared at different strain amplitudes and frequencies to an equivalent number of oscillations, the duration of shearing was adjusted accordingly with $10<t(\mathrm{~min})<40$ with higher strain amplitude experiments lasting longer times. Constant frequency experiments were accomplished by varying the strain amplitude $(\gamma=1$ and 3$)$ with a fixed frequency of $3 \mathrm{~Hz}$ and total deformation time of $5 \mathrm{~min}$. Both methods to manipulate the shear rate magnitude have been used in the literature. ${ }^{29}$ The images in Fig. 3 (collected $\sim 50 \mu \mathrm{m}$ above the coverslip) illustrate the effect that the duration of shearing has on the ordering process for a $\phi$ $=0.48$ sample sheared at $\gamma=1$ and frequency of $5 \mathrm{~Hz}$.

In Fig. 3, the images from left to right represent an unshared sample, a sample sheared for 2 min, and a sample sheared for a total of $5 \mathrm{~min}$. The insets are 2D fast Fourier transforms that show the evolution of distinct Bragg peaks in 

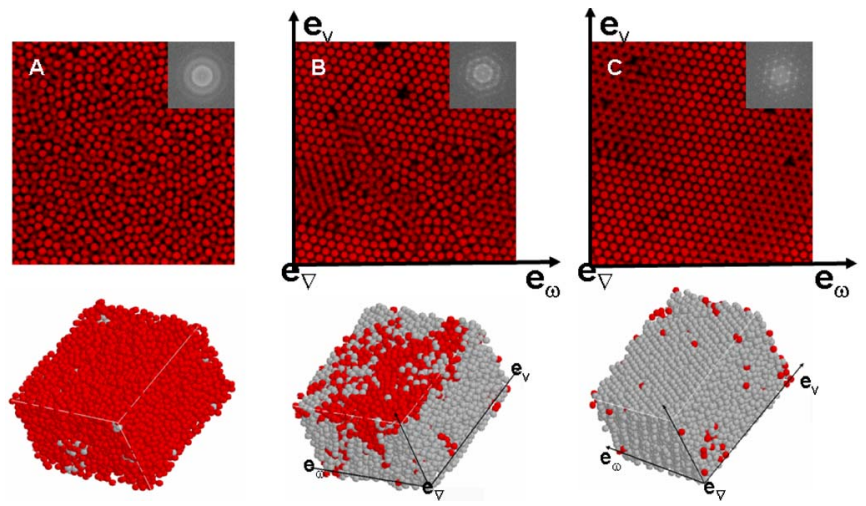

FIG. 3. 2D micrographs with FFT insets and 3D renderings illustrating the effect of time on the degree of crystallization for $\phi=0.48, \gamma=1$, and Freq $=3 \mathrm{~Hz}$. (a) Quiescent $(24 \mathrm{~h})$, (b) $t=2 \mathrm{~min}$, and (c) $t=5 \mathrm{~min}$.

the ordered samples. The 3D renderings at the bottom of the figure show the spatial degree of ordering for the entire image volume. These data document flow-induced ordering of the initially amorphous specimens and are consistent with the earlier scattering studies of Ackerson and Pusey and Ackerson. $^{28,29}$

\section{Confocal laser scanning microscopy}

The shear cell apparatus is mounted to the confocal laser scanning microscope (DM IRE2, Leica, Germany). An oil immersion of $100 \times$ objective [numerical aperture NA $=1.4$ ] was used to acquire stacks of the $2 \mathrm{D}$ images of the objective plane $(x-y$ plane $)$ at $512 \times 512$ pixel $^{2}$ resolution. These images were collected in the flow-vorticity plane of the flow. By actuating the height of the objective plane relative to the coverslip so as to traverse the gradient direction of the flow, an image volume was built up at a rate of $\sim 0.6$ frames per second. (The acquisition rate limits the image collection to after the cessation of flow.) Because of the time required to collect an image volume, the acquisition was performed immediately after the cessation of flow. Panine et al. have reported sharp jumps in crystallization immediately after the cessation of flow; ${ }^{45}$ however, the time series of 2D images here acquired immediately before and after cessation of flow did not record behavior of this kind, perhaps due to the slow dynamics in the high viscosity solvent used. Typically $\sim 3002 \mathrm{D}$ images comprise the image volume. Image volumes of approximately $30 \times 30$ $\times 20 \mu^{3}$ were obtained in about 8-9 min with both $x y$ and $z$ pixel resolutions of $61 \mathrm{~nm}$. Figures 4(a) and 4(b) show three-dimensional reconstructions of an image volume consisting of a stack of $2 \mathrm{D}$ images. The $3 \mathrm{D}$ reconstructions show the qualitative structure of a quiescent, amorphous and sheared, ordered structure $(\gamma=1$, Freq $=3, t=5 \mathrm{~min})$, respectively.

Images were collected after the flow was stopped at zero strain amplitude. Sample loading was observed to result in crystallization near the coverslip at distances below $30 \mu \mathrm{m}$ (data not shown). Thus, to exclude wall effects, images were collected above this height. A time series showed the
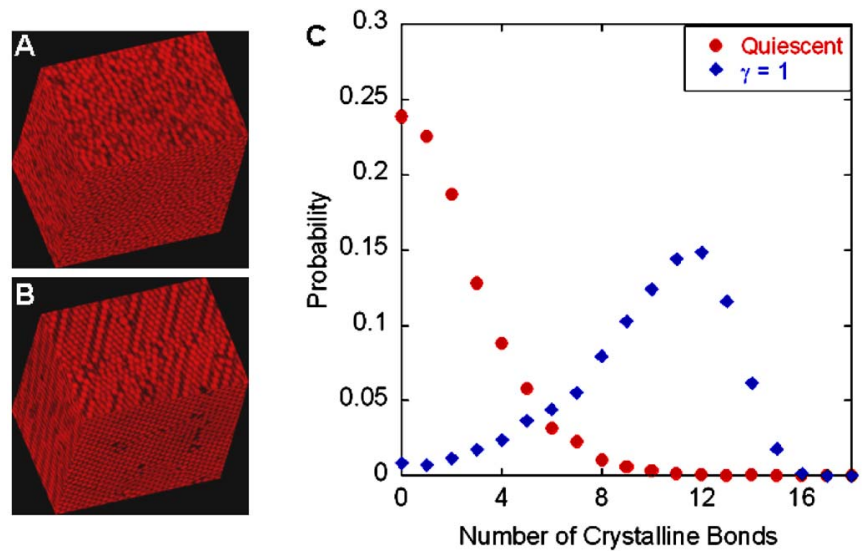

FIG. 4. Crystalline particle distribution for representative 3D amorphous and ordered image volumes. (b) Amorphous ( $\phi=0.52$, quiescent, $t=24 \mathrm{~h}$ ). (c) Ordered $(\phi=0.52, \gamma=1$, Freq $=3, t=5 \mathrm{~min})$.

samples had reached a steady state with negligible particle displacements in the high viscosity medium during the quiescent acquisition period.

To identify particle locations, we use 3D image processing algorithms adapted from Crocker and Grier. ${ }^{46}$ The 3D algorithms consist of subroutines that perform the following functions: First, convolution of the image with a Gaussian filter reduces high frequency noise. Second, particle centers are identified from the processed images by means of a local maximum criterion. Third, particle positions are refined to subpixel accuracy $( \pm 20 \mathrm{~nm} x-y, \pm 30 \mathrm{~nm} z),{ }^{44}$ by means of moments of the local intensity distribution. Once particle locations were found to suitable accuracy, bond orientational parameters were computed to differentiate particles with crystal-like and amorphous orientations. ${ }^{45}$ These calculations include the evaluation of local, bond order parameters derived from spherical harmonics,

$$
\bar{q}_{j m}(i)=\frac{\sum_{j=1}^{N_{b}(i)} Y_{l m}\left(\theta_{i j}, \phi_{i j}\right)}{N_{b}(i)} .
$$

Here the subscript $j$ designates a neighboring particle within a given radius of particle $i, N_{b}$ is the total number of neighboring particles, and $Y_{l m}\left(\theta_{i j}, \phi_{i j}\right)$ are spherical harmonics evaluated at the specified polar and azimuthal angles. ${ }^{47}$ The radius for the coordinate shell of neighboring particles was chosen near the first minimum of the $3 \mathrm{D}$ pair correlation function. Crystalline particles were identified as having eight or more neighboring particles where the coherence between bonds measured by the dot product of $\mathbf{q}_{6}(i)$ and $\mathbf{q}_{6}(j)$ exceeded $0.5 .^{48}$ Here, the $m$ th component of $\mathbf{q}_{6}(i)$ is

$$
\tilde{q}_{6 m}(i)=\frac{\bar{q}_{6 m}(i)}{\left[\sum_{m=-6}^{6}\left|\bar{q}_{6 m}(i)\right|^{2}\right]^{1 / 2}},
$$

and the dot product is

$$
\mathbf{q}_{6}(i) \cdot \mathbf{q}_{6}(j)=\sum_{m=-6}^{6} \tilde{q}_{6 m}(i) \tilde{q}_{6 m}(j)^{*},
$$

where ${ }^{*}$ denotes the complex conjugate. Figure 4(c) reports the distribution of the number of crystalline bonds per particle for the representative, quiescent, and sheared image vol- 
umes shown in Figs. 4(a) and 4(b). The figure confirms that the ten Wolde et al. criterion for local crystallinity can distinguish between quiescent and crystalline samples in our experimental system.

\section{Stacking fault analysis}

We seek to investigate the spatial distribution of stacking faults in the 3D image volumes acquired. In wall-bounded flows, scattering indicates that colloidal crystals organize into layers of close-packed planes oriented perpendicular to the wall normal direction. ${ }^{30}$ The interlayer ordering of these close-packed structures is specified by one of two possible lattice vectors. Thus, the layer registry parallel to the wall normal direction (the gradient direction) is specified by one of three possible configurations, typically denoted as $A, B$, or $C$. hcp structures consist of an " $A B A B A B$ " stacking registry. fcc crystals have an " $A B C A B C$ " stacking. The mean stacking parameter $\alpha$ has a value of 1 and 0 for perfect fcc and hcp crystals, respectively, and all possible stacking registries have intermediate stacking parameter values. Faulted crystal structures of this kind have a distinctive appearance along the (110) crystal plane. In particular, a sequence repeating every other layer (a hcp crystal) would be recognized in the (110) plane by kinks that change the close-packed direction. Figure 5 shows the distinct appearance associated with the presence of kinks in the (110) plane. The arrow highlights the location of a kink. Elliot et al. showed that the stacking parameter $\alpha$ can be computed by calculating the number of kinks for a given number of layers and applying the following expression:

$$
\alpha=1-\frac{k}{N-2},
$$

where $k$ is the number of kinks and $N$ is the total number of layers. ${ }^{34}$ We use Eq. (4) to extract the stacking parameter of the sheared crystals. This approach involved extracting eight evenly spaced cross sections from the image volumes that correspond to the (110) plane and by visual inspection, determining the number of kinks from eight different locations across each image. Each point can be characterized by a value of $\alpha$, here denoted by $\alpha(x, y)$ where $(x, y)$ denotes the location of the particular stacking sequence analyzed. By averaging over the 64 locations, a spatially averaged value of the global, stacking parameter $\langle\alpha\rangle$ for the entire image volume may be computed. We report the variation among the 64 points as the standard deviation in Table II. Furthermore, to assess the interplanar variation in the stacking registry, the layer averaged stacking parameter $\langle\alpha(z)\rangle_{L}$ is also determined by averaging the 64 values of $\alpha(x, y)$ collected in each crystal layer via the kink analysis method.

For comparison, the mean stacking parameter is also computed by a second automated method that involves direct measurement of the translational vector between different planes. For example, if a particle has a hcp $(A B A B)$ configuration, then another particle can be found at a translational vector equivalent to twice the interlayer spacing $(\sqrt{ }(2 / 3) d)$ of the crystal where $d$ is the effective colloid diameter determined from Fig. 1. To account for the deviations from a

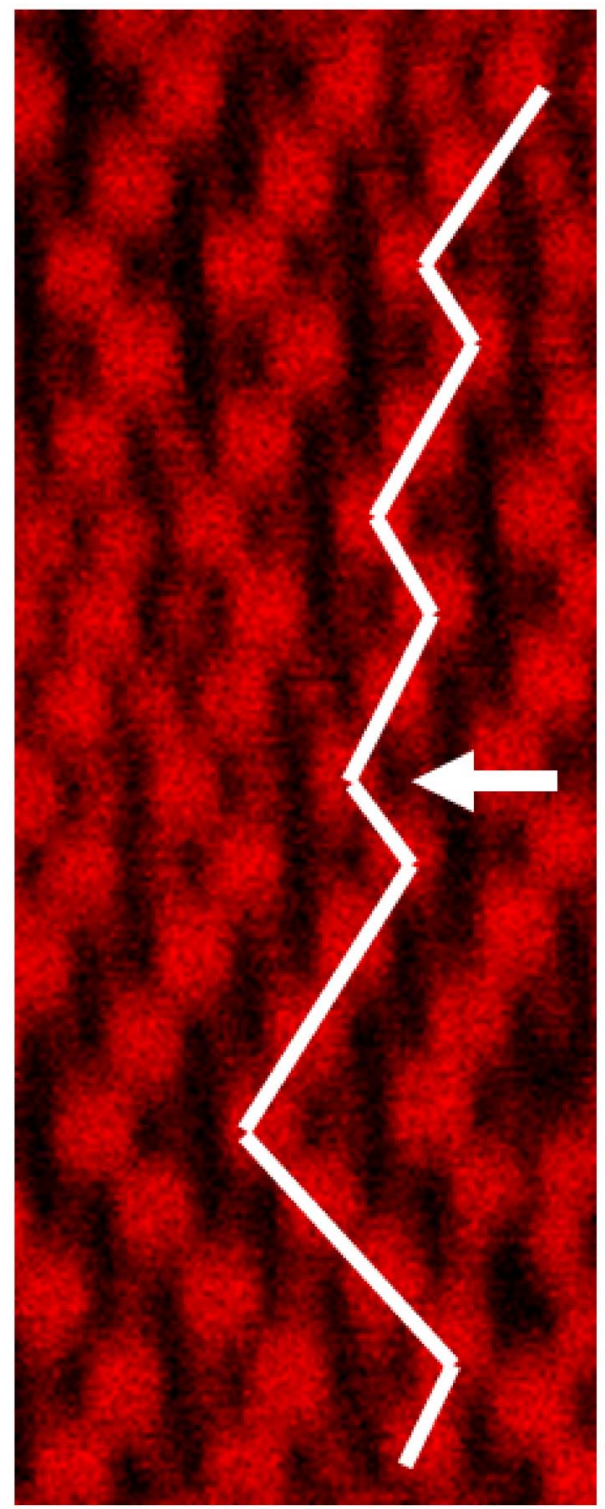

FIG. 5. (110) plane identification of kinks in a sheared colloidal crystal at $\phi=0.52, \gamma=3$, Freq $=3$.

perfect lattice due to Brownian motion, a particle was labeled hcp if another was found two layers below and within a radius of $\sqrt{ }(3) / 2 d$ that extends halfway between the next interstitial (fcc) site. fcc particles were found in a similar fashion by searching for particles within a radius from the translational vector of $\mathbf{r}=(\mathbf{i}+1 / \sqrt{ }(3) \mathbf{j}+\sqrt{ }(2 / 3) \mathbf{k}) d$.

To summarize, in the scattering literature, because the ensemble averaged structure is interrogated, a single mean

TABLE II. Summary of shear experiments. Definition of terms: $\gamma$ (strain amplitude) and $\dot{\gamma}$ (shear rate magnitude).

\begin{tabular}{cllcccc}
\hline \hline$\gamma$ & Freq & $\dot{\gamma}$ & $\mathrm{Pe}$ & $\langle\alpha\rangle$ & $\%$ crystal & $\langle\chi\rangle$ \\
\hline 1 & 4 & 4 & 8.7 & $0.96 \pm 0.07$ & 77 & - \\
3 & 1.33 & 4 & 8.7 & $0.80 \pm 0.09$ & 71 & 0.83 \\
4 & 1 & 4 & 8.7 & $0.56 \pm 0.08$ & 67 & 1.15 \\
1 & 3 & 3 & 6.5 & $0.98 \pm 0.05$ & 82 & - \\
3 & 3 & 9 & 19.5 & $0.44 \pm 0.08$ & 83 & 1.25 \\
\hline \hline
\end{tabular}


stacking parameter $\alpha$ is typically reported. Here, because our aim is to characterize heterogeneity in the crystal structure, the stacking parameter concept must be extended to accommodate the spatial resolution of the CLSM method. Thus, we have $\alpha(x, y)$, the result of a kink analysis of the layer sequence at the coordinate $(x, y),\langle a(z)\rangle_{L}$, the relative ratio of hcp and fcc structures in a particular layer a distance $z$ from the shear surface, as well as $\langle\alpha\rangle$, the global average of the mean stacking parameter in an image volume. Note that $\langle\alpha\rangle$ is the equivalent of the result that would be extracted from a scattering experiment and thus is directly comparable to earlier studies. ${ }^{28,29}$

\section{E. Degree of randomness}

In previous studies, the mean stacking parameter was the primary quantity used to characterize the stacking sequence in colloidal crystals; however, as discussed previously, this measure alone is not sufficient to determine whether the distribution of stacking faults in a colloidal crystal truly displays random statistics. However, single-parameter characterizations of randomness appear in other areas of physical chemistry, particularly in the classification of copolymer sequences. Here we adopt the measure $\chi$ used in that field. ${ }^{49-51}$ Recast in terms of quantities relevant to the stacking sequence of colloidal crystals $\chi$ is defined as

$$
\chi=\frac{[\mathrm{fcc} / \mathrm{hcp}]}{[\mathrm{fcc}][\mathrm{hcp}]},
$$

where [fcc/hcp] is the probability of finding a fcc layer followed by a hcp layer and [fcc] and [hcp] are the probabilities of finding fcc and hcp layers. This random parameter takes values between zero and two, with 0,1 , and 2 corresponding to block structure, random statistics, and alternating structure, respectively.

\section{RESULTS}

\section{A. Correspondence with previous scattering studies}

Qualitatively, we observe that execution of oscillatory shear results in the organization of initially amorphous samples into close-packed layers oriented parallel to the shear surface (Fig. 3). We find that the orientation of the close-packed layers depends on the amplitude of the applied strain. The global, mean stacking probability $\langle\alpha\rangle$ decreases with the strain amplitude. These observations are in accord with previous light and neutron scattering studies. ${ }^{29}$

By means of image processing, we extract quantitative information about the parallel layers of hexagonal closepacked [(111) plane] arrays formed by shear. Figure 6 reports the wall normal (gradient direction) number density of particles at a volume fraction $\phi=0.52$ and frequency of $3 \mathrm{~Hz}$ as a function of strain amplitude. As the strain amplitude is increased the fluctuations in number density with axial position increase. The existence of a characteristic scale for the fluctuations is apparent in the inset of the figure, where the fast Fourier transform (FFT) of the data is plotted. The maximum in the FFT power spectrum agrees well with the lattice vector expected for the close-packed layer spacing

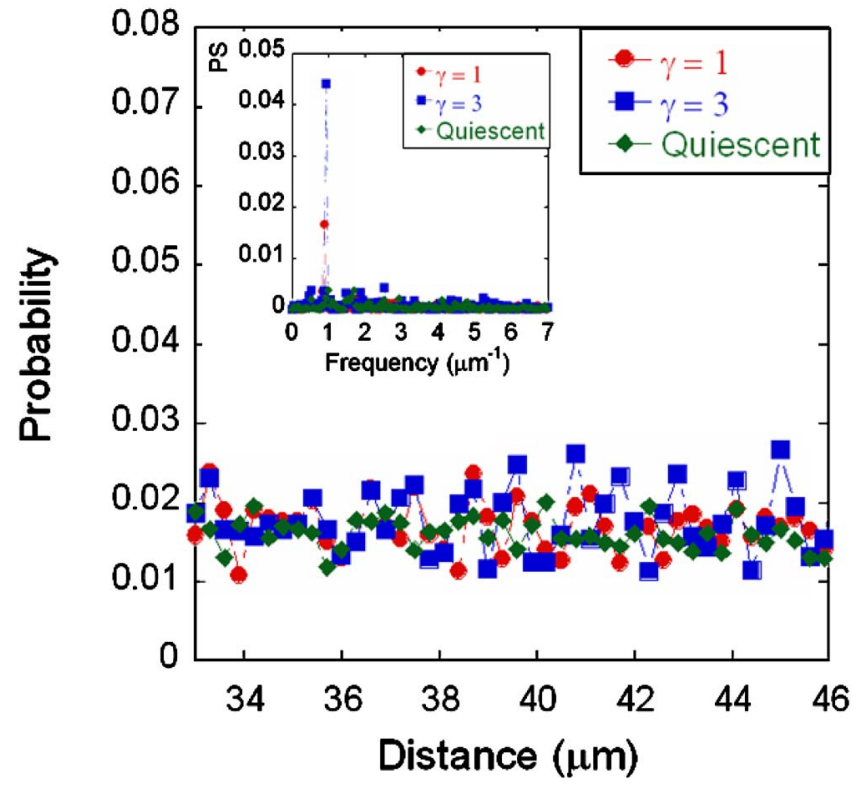

FIG. 6. Vertical number density distribution for various strain amplitudes for $\phi=0.52$, Freq $=3$. The inset is the Fourier transform of data

(Freq $=1 / \sqrt{2 / 3} d$ ) where $d$ is the effective colloid diameter. For this comparison, the characteristic particle separation $d$ is extracted from the pair potential in Fig. 1.

The variation in crystal orientation with strain amplitude can also be directly observed in the imaging plane as reported in Figs. 7(a) and 7(b). The images on the left and right are for samples sheared at $\gamma=1$ and $\gamma=3$, respectively. Both images were collected $\sim 50 \mu \mathrm{m}$ above the coverslip and are for $\phi=0.52$ sample sheared at a frequency of $3 \mathrm{~Hz}$. The insets include 2D FFTs that also show Bragg peaks consistent with the ordered structure and the shift in orientation between the two samples. The arrows highlight the particle close-packed direction. At low strain amplitudes, $\gamma=1$, the close-packed direction of the (111) layers is aligned primarily in the vorticity direction. Upon increasing the strain amplitude, $\gamma>1$, a shift in the crystal orientation that corresponds with a particle close-packed direction parallel to the flow direction occurred. The effect of strain amplitude on orientation is quantified in Fig. 7(c). The oscillations in nearest neighbor positions in the 2D layers differ in phase by $90^{\circ}$ for the two strain amplitudes.

As described earlier, our methods allow the extraction of the global, mean stacking parameter $\langle\alpha\rangle$ from the acquired image volumes and this quantity is directly comparable to
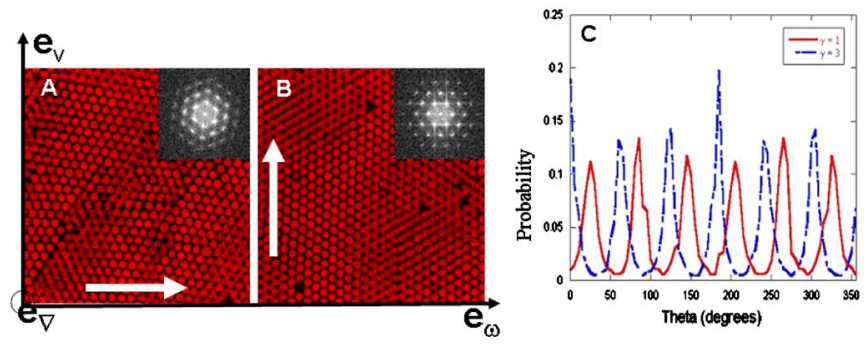

FIG. 7. Variation in crystal orientation with strain amplitude with 2D FFT insets. (a) $\gamma=1$, (b) $\gamma=3$, and (c) plot of angular distribution of first six nearest neighbors. 

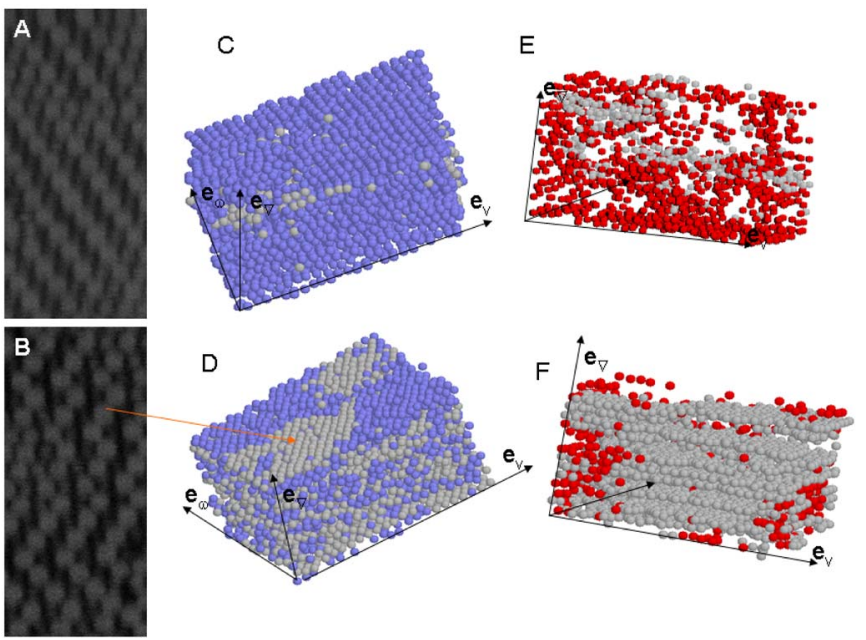

FIG. 8. 2D cross sections of (110) plane and 3D renderings of image volumes showing spatial heterogeneity of stacking registry in flow-vorticity plane and gradient direction. (a) $\gamma=1$ and (b) $\gamma=3$. Blue, gray, and red particles are fcc, hcp, and noncrystalline particles, respectively.

previous scattering measurements. Table II summarizes the results of studies in which the strain amplitude was varied at constant shear rate magnitude and at constant Peclet number. To benchmark the data, note that the quiescent samples were only $2 \%$ crystalline. Increasing the strain amplitude causes $\langle\alpha\rangle$ to decrease. The decrease from 0.97 at low strain to 0.46 at high strain is consistent with a transition from an approximately fault-free fcc crystal $(\alpha \sim 1)$ to a structure of randomly stacked layers $(\alpha \sim 0.5)$. This average analysis suggests that the number of stacking faults in the sample increases with strain amplitude.

Quantitative measurements of the degree of crystallinity were performed by differentiating between particles with crystalline and amorphous orientations per the method of Sec. II C. Table II reports the degree of crystallinity of the sheared colloidal crystals at the conditions studied. A decrease in the degree of crystallinity is observed with an increase in the strain amplitude for the constant shear rate magnitude experiments. The constant frequency experiments yield samples with nearly equal values in the degree of crystallization at the different strain amplitudes.

\section{B. Spatial heterogeneity of stacking faults}

The stacking faults that lead to the variation in global parameter $\langle\alpha\rangle$, reported in Table II are qualitatively apparent in the CLSM image volumes, as shown in the representative images of Fig. 8. Connecting particle centroids by line segments, as shown in Fig. 9(a), clearly displays the complex fault structure. In this section, we seek to understand the spatial distribution of such stacking faults. As shown in Fig. 8 , 3D renderings of the image volumes demonstrate that spatial heterogeneity is significant at large strain amplitude $(\gamma$ $=3$ ). Interestingly, the heterogeneity in the flow-vorticity plane is clearly apparent [also shown with Fig. 9(b)]. This observation is at odds with one-dimensional models commonly used to extract the mean stacking parameter from scattering. ${ }^{52,53}$ That is, in such models, interlayer registry is assumed to be uniform in the flow-vorticity plane. Figures 8
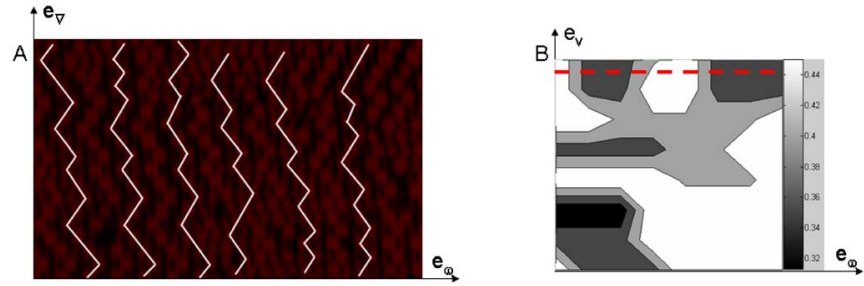

FIG. 9. (a) 2D cross section of (110) plane. (b) Contour map of $\alpha(x, y)$ values computed via kink analysis for the sample sheared at $\gamma=3$ and Freq $=3 \mathrm{~Hz}$ illustrating the spatial heterogeneity in the flow-vorticity plane and gradient direction. Dashed red line corresponds to location of cross section in (a).

and 9 suggest that the one-dimensional (1D) assumption is too restrictive to model the behavior of close-packed colloidal crystallization.

To assess the degree to which flow-vorticity plane variations in stacking faults contribute to the variations in $\langle\alpha\rangle$ reported in Table II, we analyzed the spatial heterogeneity by a number of methods. First, in Fig. 10, we report the axial variation in the stacking of each layer $\langle\alpha(z)\rangle_{L}$. Here, a uniformly fcc layer at a distance $z$ from the coverslip would have $\langle\alpha(z)\rangle_{L}=1$. Independent of $z$, a uniformly hcp layer would have $\langle\alpha(z)\rangle_{L}=0$. Independent of $z,\langle\alpha(z)\rangle_{L}=0.5$ indicates that half the particles in that particular $(x, y)$ layer are part of a fcc structure, while half the particles are part of a hcp structure. If the assumptions of a fully one-dimensional model of stacking registry (such as Loose and Ackerson or Guinier ${ }^{52,53}$ ) were valid, then the only possible values of points on Fig. 10 would be zero and one. For the sample subjected to the strain of one, this assumption is nearly correct as we observe many defect-free fcc layers with $\langle\alpha(z)\rangle_{L}$ $=1$.

At high strain amplitude, the layer structure is clearly laterally inhomogeneous since $\langle\alpha(z)\rangle_{L}$ often takes values between zero and one. We observe a number of planes where

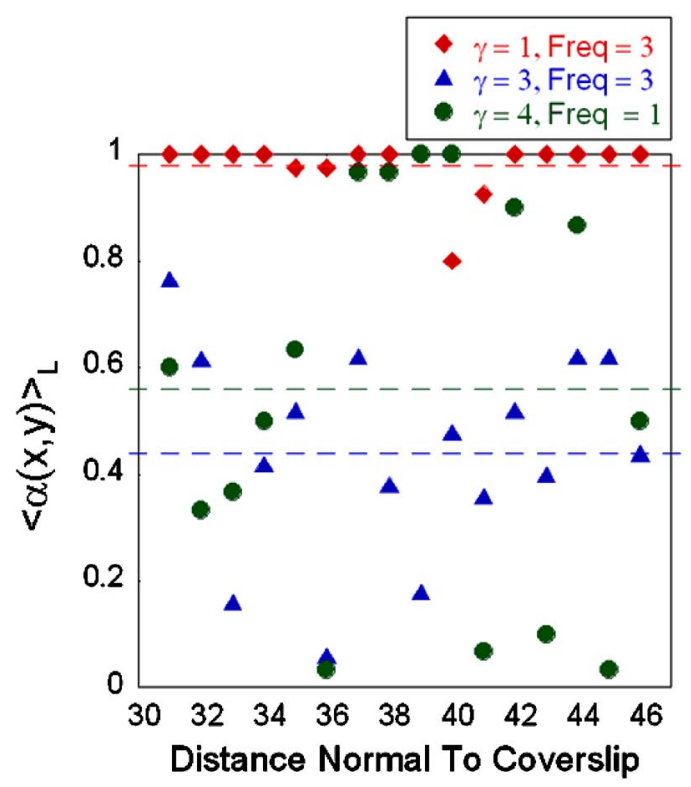

FIG. 10. Axial variation in stacking registry of $\phi=0.52$ sheared crystals at various strain amplitudes and frequencies. Dashed lines are the mean value $\langle\alpha(z)\rangle_{L}$ averaged over all layers. 
$\langle\alpha(z)\rangle_{L}$ is nearly zero. Such stacking faults extend laterally across the entire image volume. These faulted hcp layers are interspersed among polycrystalline layers that consist of a mixture of hcp and fcc regions which leads to an intermediate value of $\langle\alpha(z)\rangle_{L}$ for that particular plane. The sample subjected to $\gamma=4$ displays the largest variation in $\langle\alpha(z)\rangle_{L}$. Figure 10 shows that the samples subjected to strain amplitudes of 3 and 4 have comparable stacking parameters values; however, significant variations in the arrangement of stacking faults are observed. The deviations from $\langle\alpha(z)\rangle_{L}$ at a number of points are then due to a number of hop areas. However, these layers, since $\langle\alpha(z)\rangle_{L}<1$, do not extend laterally throughout the whole specimen.

As previously mentioned, the quantitative analysis of the stacking registry above is represented qualitatively in Fig. 8 with 2D images of the (110) plane and 3D renderings for samples from the constant frequency experiments (details in Table II). The volumes show qualitative variations in the stacking registry in both the flow gradient and flow-vorticity experiments. The images at the top correspond to $\gamma=1$ and image on the bottom to $\gamma=3$. hcp, fcc [Figs. 8(c) and 8(d)], and amorphous [Figs. 8(e) and 8(f)] particles are identified as gray, blue, and red, respectively. For the sample subjected to $\gamma=1$, the variation in the stacking registry consists mainly of small faulted regions. The rendering for $\gamma=3$ illustrates the presence of polycrystalline layers in the flow-vorticity plane, in good correspondence with the results of Fig. 10.

To further identify the degree of spatial heterogeneity of stacking faults, we selected 64 points in the flow-vorticity plane $(x y)$ and performed a kink analysis (in the axial, gradient direction) at those points. Typically, sequences of crystalline particles completely extend through the imaged volume of the colloidal crystal (approximately 18 layers). Kinks define each layer as fcc or hcp, as per Eq. (1), and from this analysis we computed $\alpha(x, y)$ for the $\gamma=3$ sample, as reported in the contour map of Fig. 9(b). The spatial variation in $\alpha(x, y)$ is striking: Although $\langle\alpha\rangle$ for this sample is 0.46 , we see that over the $31 \times 31 \mu \mathrm{m}^{2}$ surface area of the colloidal crystal $\alpha(x, y)$ varies from 0.31 to 0.5 . From Fig. 9(b), we estimate the scale of lateral inhomogeneity to be $\sim 5-10$ particle diameters for these conditions $(\gamma=3)$.

Thus from Figs. 8-10, we learned that the spatial heterogeneity of stacking faults is prevalent in the flow-vorticity plane and this is one source of $\langle\alpha\rangle \sim 0.5$ in the high strain amplitude region. We now address the complementary question of the distribution of stacking faults in the gradient direction. (For example, a crystal with $\langle\alpha\rangle=0.5$ could equally well be comprised of a hcp crystal stacked on top of a fcc crystal or alternating thin layers of the two structures). In Fig. 11(a), we plot the number of fcc to hcp transitions in the 18 crystal layers and in Fig. 11(b) we plot the number of hcp to fec transitions.

First, to compare our results with random layer ordering, we build random layer sequences by randomly selecting one of the two possible lattice vectors possible for close-packed layers. In a way that was exactly analogous to the experiments we produce 64 such random sequences each of 18 layers. The sequence of random lattice vector translations
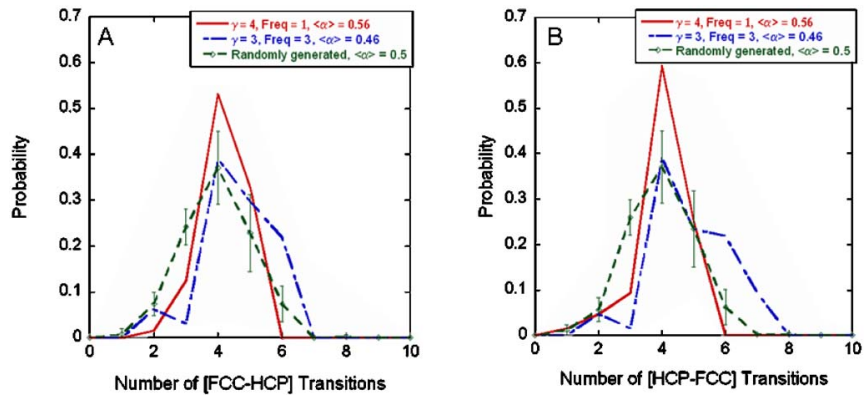

FIG. 11. The number of layer transitions in vertical stacking sequence for samples with comparable mean stacking parameter.

was converted to the traditional indexing for close-packed layers $(A B C B A \cdots)$ and data analysis performed as in the experiments. Results are plotted in Figs. 11(a) and 11(b). In Fig. 11, we observe that the distributions for both types of transitions are peaked at a value of 4 (that is, within the 18 layers the most probable number of transitions is 4). For the eighteen layer sequences observed, this magnitude of transitions indicates a structure with fcc and hcp layers interspersed together and having relatively short sequences of each layer. Interestingly, we also observe differences in the width and magnitude of the distributions for the experimentally observed microstructures from that of the randomly generated case. In the next section, we address the randomness of these distributions.

\section{Randomness}

Although $\langle\alpha\rangle$ (Table II) of the high amplitude sheared colloidal crystals is consistent with the random sliding layer model of Ackerson and co-workers, the probability distributions of Fig. 11 suggest the possibility of nonrandom stacking of fcc and hep layers since the experimental distributions vary from the randomly generated case. We address this point in Fig. 12 by computing the randomness parameter $\chi$ defined in Sec. II E. We also compute the value of the randomness parameter $\chi$ for each randomly generated series and plot the distribution of chi values for the 64 series. The results are reported in Fig. 12 and indicate that the distribution

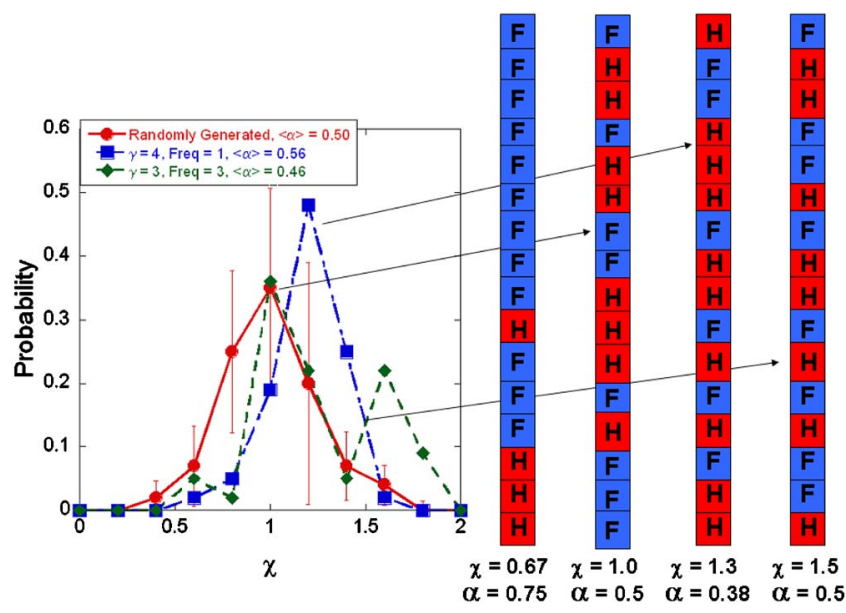

FIG. 12. Randomness parameter distributions and illustrations of various stacking sequences. 
of hcp and fcc layers does not agree with that extracted for the corresponding random process. In this context, a random process should yield a distribution of fcc and hcp layer thicknesses with the peak centered at a value of $\chi=1$ as evidenced by the random layer simulations. Here the thickness of the colloidal crystals structures we analyze are thin $(\sim 18$ layers, as would be typical of applications ${ }^{3}$ ).

A truly random layering will yield a distribution with mean $\chi=1$. As the number of layers analyzed increases, the width of the distribution decreases toward a limit of a strongly peaked distribution centered around 1. For the 18 layers characteristic of the experiments, we see from Fig. 12 that the theoretical random $\chi$ distribution is broad: extending from about $0.5<\chi<1.5$. On the other hand, the behavior of the measurements is different. First, as previously reported in Table II and shown as well in Fig. 12, for the samples with $\langle\alpha\rangle \sim 0.5$, the mean value of $\chi$ is shifted to values significantly greater than one. Interestingly, this shift indicates that these samples have a preference toward alternating structure relative to random structure. This shift is a principal finding of this study.

\section{CONCLUSIONS}

We have characterized the 3D stacking fault structure of colloidal crystals formed with the application of an oscillatory flow field. To complement the extensive understanding of sheared crystals obtained from prior scattering studies, we have used the direct visualization of the stacking registry via CLSM to obtain measurements of the spatial heterogeneity of the stacking fault structure in both the gradient direction and flow-vorticity plane. These results demonstrate crystals sheared at large strain amplitudes $(\gamma>3)$ that would be predicted by the assumption of random transitions between the close-packed that lattice vectors have in fact a stacking structure consisting of nonrandom, short alternating layers of fcc and hcp crystal planes that persist of order $\sim 5-10$ particle diameters in the lateral direction. The heterogeneous and nonrandom microstructures seen in this study are significant in that they suggest that theories describing the deformation of shear-induced crystals at large strain amplitudes may need to be more detailed than the one-dimensional sliding layer mechanisms originally proposed. ${ }^{50,51}$

Finally, the microstructure of shear-induced colloidal crystals in this study is also of technological interest because recent studies have proposed the application of shear to assemble colloidal crystals for photonic applications. ${ }^{14,54}$ The short, alternating stacking sequences formed are thus relevant to the ultimate optical properties of crystals that might be fabricated from these materials. ${ }^{12}$

\section{ACKNOWLEDGMENTS}

We thank and recognize Reginald Rogers for the characterization of the pair potential, undergraduates Tim Lewer and Amy Herzog for their efforts in the synthesis of colloidal particles, and also the funding supports from Rackham Graduate School (University of Michigan), GEM, NOBCChE, NSF (CTS-0093076), and NASA (NNC04GA66G).
${ }^{1}$ P. N. Pusey and W. van Megan, Nature (London) 320, 340 (1986).

${ }^{2}$ S. L. Kuai, V. V. Truong, A. Hache, and X. F. Hu, J. Appl. Phys. 96, 5982 (2004).

${ }^{3}$ Y. Lee and P. V. Braun, Adv. Mater. (Weinheim, Ger.) 15, 563 (2003).

${ }^{4}$ S. I. Matsushita, T. Miwa, D. A. Tryk, and A. Fujishima, Langmuir 14, 6441 (1998).

${ }^{5}$ V. J. Anderson and H. N. W. Lekkerkerker, Nature (London) 416, 811 (2002).

${ }^{6}$ A. P. Gast and W. B. Russel, Phys. Today 51(12), 24 (1998)

${ }^{7}$ W. C. K. Poon, J. Phys.: Condens. Matter 14, R859 (2002).

${ }^{8}$ M. D. Haw, W. C. K. Poon, and P. N. Pusey, Phys. Rev. E 57, 6859 (1998).

${ }^{9}$ S. Pronk and D. Frenkel, J. Chem. Phys. 120, 6764 (2004).

${ }^{10}$ P. Schall, I. Cohen, D. A. Weitz, and F. Spaepen, Science 305, 1944 (2004).

${ }^{11}$ V. N. Astratov, Y. A. Vlasov, A. V. Baryshev, A. A. Kaplyanskii, O. Z. Karimov, and M. F. Limonov, Phys. Rev. E 61, 5784 (2000).

${ }^{12}$ V. Yannopapas, N. Stefanou, and A. Modinos, Phys. Rev. Lett. 86, 4811 (2001).

${ }^{13}$ A. van Blaaderen, MRS Bull. 29, 85 (2004).

${ }^{14}$ T. Sawada, Y. Suzuki, A. Toyotama, and N. Iyi, Jpn. J. Appl. Phys., Part 2 40, L1226 (2001).

${ }^{15}$ P. T. Korda and D. G. Grier, J. Chem. Phys. 114, 7570 (2001).

${ }^{16}$ J. P. Hoogenboom, D. Derks, P. Vergeer, and A. van Blaaderen, J. Chem. Phys. 117, 11320 (2002).

${ }^{17}$ H. M. Lindsey and P. M. Chaikin, J. Chem. Phys. 76, 3774 (1982).

${ }^{18}$ S. Pronk and D. Frenkel, J. Chem. Phys. 110, 4589 (1999).

${ }^{19}$ S. C. Mau and D. A. Huse, Phys. Rev. E 59, 4396 (1999).

${ }^{20}$ Z. Cheng, J. Zhu, W. B. Russel, W. V. Meyer, and P. Chaikin, Appl. Opt. 40, 4146 (2001)

${ }^{21}$ W. K. Kegel and J. K. G. Dhont, J. Chem. Phys. 112, 3431 (2000).

${ }^{22}$ P. N. Pusey, W. van Megen, P. Bartlett, B. J. Ackerson, J. G. Rarity, and S. M. Underwood, Phys. Rev. Lett. 63, 2753 (1989).

${ }^{23}$ J. Zhu, M. Li, R. Rogers, W. Meyer, R. H. Ottewill, W. B. Russel, and P. M. Chaikin, Nature (London) 387, 883 (1997).

${ }^{24}$ B. O'Malley and I. Snook, Phys. Rev. Lett. 90, 085702 (2003).

${ }^{25}$ V. C. Martelozzo, A. B. Schofield, W. C. K. Poon, and P. N. Pusey, Phys. Rev. E 66, 021408 (2002).

${ }^{26}$ L. B. Chen, B. J. Ackerson, and C. F. Zukoski, J. Rheol. 38, 193 (1994).

${ }^{27}$ L. B. Chen, C. F. Zukoski, B. J. Ackerson, H. J. M. Hanley, G. C. Straty, J. Barker, and C. J. Glinka, Phys. Rev. Lett. 69, 688 (1992).

${ }^{28}$ B. J. Ackerson and P. N. Pusey, Phys. Rev. Lett. 61, 1033 (1988).

${ }^{29}$ B. J. Ackerson, J. Rheol. 34(4), 553 (1990).

${ }^{30}$ J. Vermant and M. J. Solomon, J. Phys.: Condens. Matter 17, R187 (2005).

${ }^{31}$ A. D. Dinsmore, E. R. Weeks, V. Prasad, A. C. Levitt, and D. A. Weitz, Appl. Opt. 40, 4152 (2001).

${ }^{32}$ A. Mohraz and M. J. Solomon, Langmuir 21, 5298 (2005).

${ }^{33}$ M. J. Solomon and M. Kogan, "Confocal Optical Microscopy" in Encyclopedia of Condensed Matter Physics, edited by G. Bassani, G. Liedl, and P. Wyder (Elsevier, New York, 2005), pp. 229-235.

${ }^{34}$ M. S. Elliot, B. T. F. Bristol, and W. C. K. Poon, Physica A 235, 216 (1997).

${ }^{35}$ I. Cohen, T. G. Mason, and D. A. Weitz, Phys. Rev. Lett. 93, 046001 (2004).

${ }^{36}$ R. M. Amos, J. G. Rarity, P. R. Tapster, and T. J. Shepherd, Phys. Rev. E 61, 2929 (2000).

${ }^{37}$ C. P. Royall, M. E. Leunissen, and A. van Blaaderen, J. Phys.: Condens. Matter 15, S3581 (2003).

${ }^{38}$ L. Antl, J. W. Goodwin, R. D. Hill, R. H. Ottewill, S. M. Owens, and S. Papworth, Colloids Surf. 17, 67 (1986).

${ }^{39}$ A. I. Campbell and P. Bartlett, J. Colloid Interface Sci. 256, 325 (2002).

${ }^{40}$ C. Pathmamanoharan, K. Groot, and J. K. G. Dhont, Colloid Polym. Sci. 275, 897 (1997).

${ }^{41}$ P. D'Haene, J. Mewis, and G. G. Fuller, J. Colloid Interface Sci. 156, 350 (1993).

${ }^{42}$ N. J. Wagner, J. Mewis, and L. Krishnamurthy, Abstr. Pap. - Am. Chem. Soc. 229, U652 (2005).

${ }^{43}$ T. O. Pangburn and M. A. Bevan, J. Chem. Phys. 123, 174904 (2005).

${ }^{44}$ P. Varadan and M. J. Solomon, Langmuir 19, 509 (2003).

${ }^{45}$ P. Panine, T. Narayanan, J. Vermant, and J. Mewis, Phys. Rev. E 66, 022401 (2002)

${ }^{46}$ J. C. Crocker and D. G. Grier, J. Colloid Interface Sci. 179, 298 (1996).

${ }^{47}$ U. Gasser, E. R. Weeks, A. Schofield, P. N. Pusey, and D. A. Weitz, 
Science 292, 258 (2001).

${ }^{48}$ P. R. ten Wolde, M. J. Ruiz-Montero, and D. Frenkel, J. Chem. Phys. 104, 9932 (1996).

${ }^{49}$ D. I. Choi, E. H. Yeom, M. Park, J. K. Kim, and B. C. Kim, J. Appl. Polym. Sci. 93, 2082 (2004).

${ }^{50}$ R. Y. F. Liu, Y. S. Hu, M. R. Hibbs, D. M. Collard, D. A. Schiraldi, A. Hiltner, and E. Baer, J. Polym. Sci., Part B: Polym. Phys. 41, 289 (2003).
${ }^{51}$ J. L. Koenig, Chemical Microstructure of Polymer Chains (Wiley, New York, 1980).

${ }^{52}$ W. Loose and B. J. Ackerson, J. Chem. Phys. 101, 7211 (1994).

${ }^{53}$ A. Guinier, X-Ray Diffraction in Crystals, Imperfect Crystals, and Amorphous Bodies (Dover, New York, 1994).

${ }^{54}$ O. Vickreva, O. Kalinina, and E. Kumacheva, Adv. Mater. (Weinheim, Ger.) 12, 110 (2000). 